\title{
An investigation of highly accurate and precise robotic hole measurements using non-contact devices
}

\author{
Zahid Usman ${ }^{2, \star}$, Radmehr P. Monfared ${ }^{1}$, Niels Lohse $^{1}$, and Michael R. Jackson ${ }^{1}$ \\ 1 EPSRC Centre for Innovative Manufacturing in Intelligent Automation, School of Mechanical and Manufacturing Engineering, \\ Loughorough University, UK \\ 2 Institute for Advanced Manufacturing and Engineering, Faculty of Engineering, Environment and Computing, Coventry \\ University, UK
}

Received: 22 January 2016 / Accepted: 10 April 2016

\begin{abstract}
Industrial robots arms are widely used in manufacturing industry because of their support for automation. However, in metrology, robots have had limited application due to their insufficient accuracy. Even using error compensation and calibration methods, robots are not effective for micrometre $(\mu \mathrm{m})$ level metrology. Non-contact measurement devices can potentially enable the use of robots for highly accurate metrology. However, the use of such devices on robots has not been investigated. The research work reported in this paper explores the use of different non-contact measurement devices on an industrial robot. The aim is to experimentally investigate the effects of robot movements on the accuracy and precision of measurements. The focus has been on assessing the ability to accurately measure various geometric and surface parameters of holes despite the inherent inaccuracies of industrial robot. This involves the measurement of diameter, roundness and surface roughness. The study also includes scanning of holes for measuring internal features such as start and end point of a taper. Two different non-contact measurement devices based on different technologies are investigated. Furthermore, effects of eccentricity, vibrations and thermal variations are also assessed. The research contributes towards the use of robots for highly accurate and precise robotic metrology.
\end{abstract}

Keywords: Robotic metrology, non contact hole measurement

\section{Introduction}

Robots have been widely used in automation of various manufacturing processes such as assembly, drilling, painting, and material handling [1-4]. Robots are also applicable in automation of metrology. This is particularly true for aircraft industry because the large, complex and adaptable work volumes of robots are suitable for aircraft parts $[2,4,5]$. However, robots have had limited use in high accuracy metrology because of their limited accuracy. Industrial robots have good repeatability i.e. from $\pm 50 \mu \mathrm{m}$ to $\pm 300 \mu \mathrm{m}[1,3]$, yet their positional accuracy can have errors of several millimetres [6]. Applications in aircraft industry have had and continue to require accuracy and precision up to micrometer " $\mu \mathrm{m}$ " $[2,7-10]$. Robots have not been effectively used for applications requiring up to $\mu \mathrm{m}$ accuracy and precision even with latest error compensation technologies.

One such application is hole measurement in the aircraft industry. Aircraft parts are large, complex and require thousands of holes to be drilled which need to be highly accurate $[11,12]$. These holes are difficult to mea-

^ Correspondence: ac1095@coventry.ac.uk sure and verify using conventional metrology equipment such as Co-ordinate Measuring Machines (CMMs). It is often not possible or desirable to bring parts to specialised CMMs or metrology labs $[13,14]$. Industrial robots would be more suitable to facilitate such measurements because they could move more easily around the parts during the normal manufacturing process and reach holes at many different locations and orientations. Industrial robots can also be mounted on movable platforms such as linear slides for enhanced work volumes. However, the work reported in this paper only focuses on industrial robot on static platforms.

For robotic hole inspection, metrology devices need to be integrated with robots. To choose an appropriate device, the measurement challenges should be considered. For example, holes to be measured can be hidden/inaccessible, positioned at oblique angles, and have small radii and tapers. The required measurement parameters can be diameter, roundness and surface roughness (Ra). The holes can be located on a large aircraft component that is difficult to be moved for measurements.

Conventional contact based metrology equipment such as touch probes and various contact based gauges cannot be used effectively to measure all those holes due to 
accessibility (to undercut holes) and capability (to measure Ra) issues. Moreover, contact based devices with up to $\mu \mathrm{m}$ accuracy require a manipulation mechanism with high accuracy in positioning which is not achievable using large industrial robots. On the other hand, non-contact devices can access hidden holes and measure without requiring to be positioned to $\mu \mathrm{m}$ accuracy. Moreover, in addition to geometric measurements, non-contact devices can also identify and measure surface anomalies, hole profile and surface roughness (Ra). Therefore, non-contact measurement devices are, potentially, more suitable for integration on robots.

However, the integrated use of such device with robots has not been investigated for hole measurement. The robotic manipulation of the device could introduce errors and inconsistencies in the measurements which may not occur in non-robotic static and controlled environments. This leads to the research question investigated in this paper: Can high accurate and precise hole measurement be achieved with non-contact measurement devices mounted on industrial robots?

This investigation contributes to the wider knowledge by providing an understanding of the effects robot movements have on highly accurate and precise hole measurement. Because $\mu \mathrm{m}$ accuracy and precision is considered, effects of vibrations and thermal variations have also been investigated.

The paper is structured as follows. Section 2 provides a review of the related work on error compensation methods for robots and suitable metrology devices for robot. The following sections explain the experimental design and setup. The next sections reports on the experimental investigation into acceptable range of probe eccentricity and the precision of robotic measurement in comparison with static measurements. Section 4.4 reports on the main set of experiments by measuring various hole parameters on a robot. Finally the results are discussed in details and useful conclusions are drawn.

\section{Relevant work}

Positional inaccuracies of robots are commonly compensated through large volume metrology and calibration systems such as vision trackers, laser trackers $[15,16]$ and other closed loop feedback systems $[3,12,17]$. Other methods include part localisation, model-based error compensation and sensor-based error compensation. Part localisation uses various part features to generate coordinate frames. These frames enhance the accuracy of robots with respect to parts $[18,19]$. Model-based error compensation and sensor-based error compensation approaches use error models and sensor feedback to correct the position of a robot $[20,21]$. Using such methods and techniques, the accuracy of robots can be brought to below $100 \mu \mathrm{m}[22]$ for smaller and closed chain robots. The accuracy of larger and open chain industrial robots is still multiple $100 \mu \mathrm{m}$. Such levels of accuracy are not sufficient for the required measurement accuracies for most applications [8-10].
The mobile devices e.g. optical $\mathrm{CMM}^{1}$ [15] and other similar probes and equipment are not suitable for accessing hidden holes because of contact based measurements. In addition, the measurement processes based on CMMs and touch based systems are slow [14,23]. Moreover, the accuracy required to position probes for contact measurement is not achievable even with external error compensation. Therefore, the use of CMM and other similar equipment is excluded from this research.

One of the common methods for inspecting holes in aircraft industry is to use plug gauges such as Go/NoGo gauges. In this method, a gauge is required for each hole diameter. Use of such gauges is labour intensive and leads to protracted inspection times [7]. Such gauges cannot measure and can only sentence inspected holes as pass or fail. Electro mechanical gauges e.g. strain gauges can also be employed for inspecting hole profiles. However, it is difficult to measure smaller and hidden holes using strain gauges and the measurement process is slow and difficult due to their inherited mechanical complexity and accessibility issues [7]. Therefore, such gauges and other contact based equipment are not effective for measuring the hidden holes and therefore not considered.

Using the latest non-contact measurement devices, both the issues of accessibility as well as more detailed surface measurements can be overcome. Non-contact measurement systems facilitate rapid inspection and the issues of vibrations can be resolved through them [23-26]. They can also facilitate measurement of surface properties and internal features.

One of the rapidly developed non-contact inspection method is machine vision [24]. Despite having high speed the accuracy of vision-based inspection is limited [24]. Moreover, standard machine vision solutions offer measurement of externally visible features and not internal and hidden features in holes.

Optical, capacitive and pneumatic non-contact method can also be used for inspecting hole geometry, form and surface features. For example, fiber-optic based non-contact methods have been used for internal measurement [25]. However, their use has been limited to very small features and they have not been explored for measuring geometry and form of holes [25]. Capacitive sensors have been effectively used for measuring small hole diameters with high accuracy [26, 27]. However, the approach has been limited to micro holes and had large errors for blind holes [28].

Eddy current probes equipped with rotation mechanism have been used for highly accurate and precise hole measurement as shown by [29]. The system could not measure form and geometry of hole but can do surface inspection.

One of the most advanced non-contact hole measurement systems is based on confocal principle [30]. The confocal device integrated with a rotation mechanism makes

\footnotetext{
${ }^{1}$ Optical CMM is a touch-based hand held probe and should not be understood as a non-contact measurement system. The optics are used to track the position and orientation of the probe (Nikon, 2014 accessed 19/07/2014).
} 


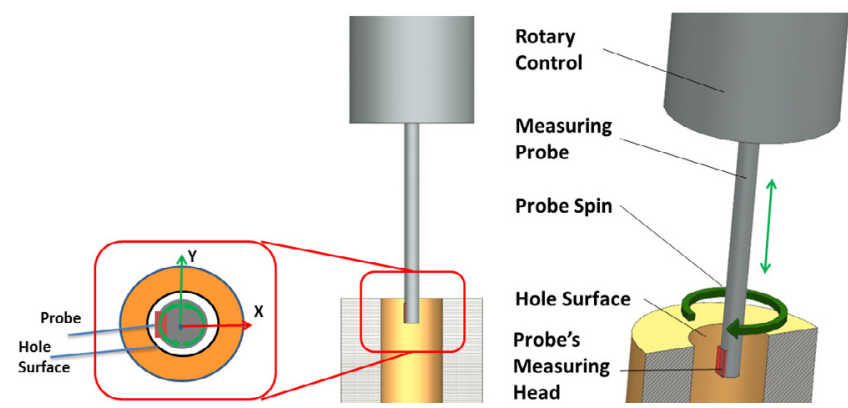

Fig. 1. Representation of confocal and low coherence interferometric hole inspection devices.

it possible to measure hole geometry and form in detail [31] as shown in Figure 1. It can be used for highly accurate and precise hole measurements as well as for scanning holes $[31,32]$.

Another non-contact hole measurement system that provides $\mu \mathrm{m}$ level accuracy and precision is based on low coherence interferometry called the Low Coherence Distance Scanning (LCDS) [33]. The LCDS system integrated with a rotatory mechanism makes it possible to inspect hole features [33,34] as shown in Figure 1. Basic hole geometry e.g. Diameters and Roundness as well as Ra surface roughness can be measured at high speed. A provision of linear movement along central axis also enables the device to scan hole surface to measure internal features. High accuracy, precision, and surface inspection capabilities in addition to measuring basic hole geometry at high speed makes LCDS and confocal devices suitable for robotic hole inspection.

Therefore, the confocal and LCDS devices have been chosen for an investigation into highly accurate and precise robotic hole measurement. The research focused to determining: (i) the accuracy and precision of robotic hole measurements at micrometre accuracy by studying the impact of robot motions on the measurement; and (ii) the ability to scan and measure internal features such as tapers and hidden holes.

\section{Experimental design}

\subsection{Stages of experimental investigation}

When high accuracy metrology devices are used on industrial robots, a number of factors that are otherwise controlled in metrology rooms, need to be considered. Therefore, a set o-f enabling experiments have been designed in addition to the main experiments to understand the effect of those factors.

The experimental investigation has been divided into two stages as shown in Figure 2. The first stage consists of the enabling experiments. These include experiments to understand the effects of (1) the eccentricity of the probe; (2) vibration; and (3) thermal variations. The second and main stage of experiments consists of two sets of experiments. These experiments have been designed with respect

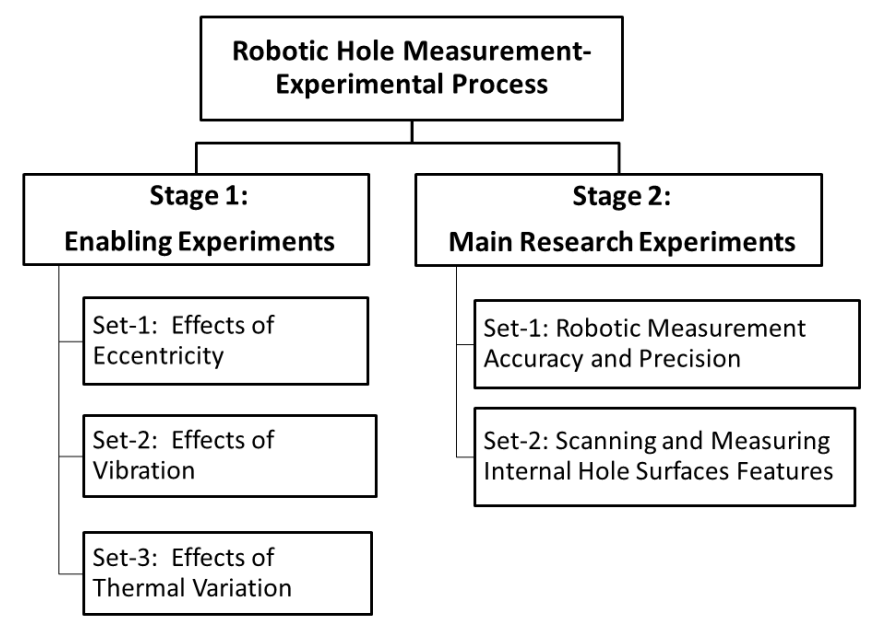

Fig. 2. Stages of experimental investigation.

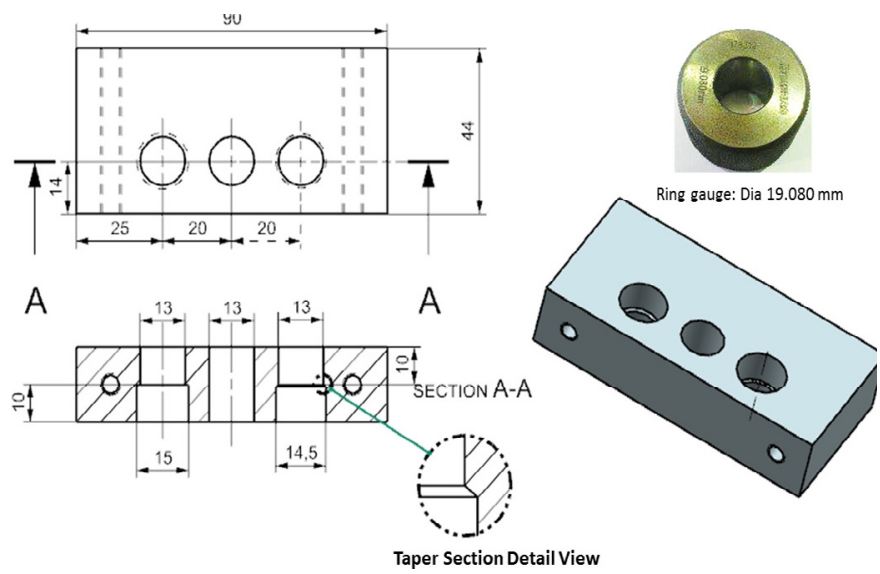

Fig. 3. The test part with straight hole, stepped hole and tapered hole.

to the two main research challenges i.e. (1) highly accurate and precise robotic hole measurement and (2) ability to scan and measure internal hole features using a noncontact device on a robot. The experimental setup and experiments are detailed in the following sections.

\subsection{Artefact design}

An important element of experimental setup is the test artefact that has been designed to represents the research challenges. The artefact was designed to have (1) a simple straight hole; (2) a hidden undercut hole (3) an undercut tapered hole as shown in Figure 3 . The diameter range from $13 \mathrm{~mm}$ to $15 \mathrm{~mm}$ was chosen because it was within the measureable range of the devices. The three holes and their features were measured during the tests in the first two sets of experiments in stage 2 . It is also important to mention here that whilst there are no blind holes in the artefact the selected hole measurement devices are capable of measuring blind holes. 


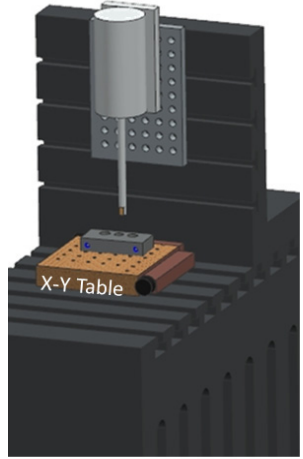

(a)
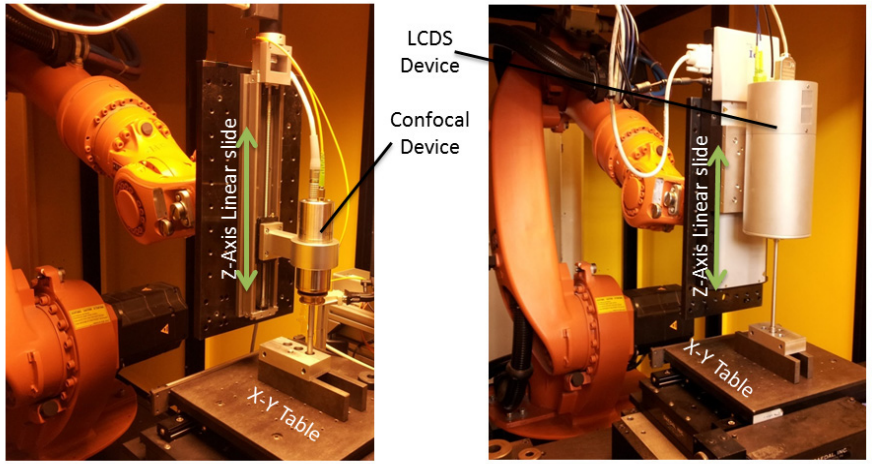

(b)

Fig. 4. Experiment setup (a) static platform (b) robotic platforms.

\subsection{The experiment setup}

The experiments involve comparing the measurement taken on a static platform with the ones taken using robotic platforms. The measurements from different the two non-contact measurement devices (Confocal Device and the LCDS device) are also to be compared. Therefore, the experimental setup was designed to facilitate such measurements. Two main types of setups were designed for the experiments as shown in Figure 4 i.e. static platform (Fig. 4a) and the robotic platform (Fig. 4b). Both the setups involved the use of an XY-table and a test artefact as shown in Figure 4. The movement of the probe along the vertical Z-Axis (i.e. into the holes) was controlled by the linear slides in both setups. The static platform consisted of an angle plate whereas the movable platform was a six axis industrial robotic arm (KUKA- KR-16). The XY-table was used to adjust the initial position of the part under the probes. To facilitate comparison, for each stage of the experimentation the device was mounted on the angle plate and then the robotic arm.

For calibration as well as actual measurements, the probe needed to be positioned in the centre of the hole. Hence, the central axis of the hole and probe needed to be aligned. The part was positioned on the XY table and centred with respect to the probe. This step was only required during the setup.

The probe required calibration before being used. A standard ring gauge of known diameter was used for the calibration purposes.

\section{Experimental results}

\subsection{Stage 1, Set 1: Determining the acceptable range of eccentricity}

The measurement probe requires to be precisely positioned at the centre of the holes for accurate readings. Beyond a certain eccentricity the measurements become sporadic and unreliable. Therefore, the deviation from the ideal centre of the hole within which the probes can still be used with confidence was determined.

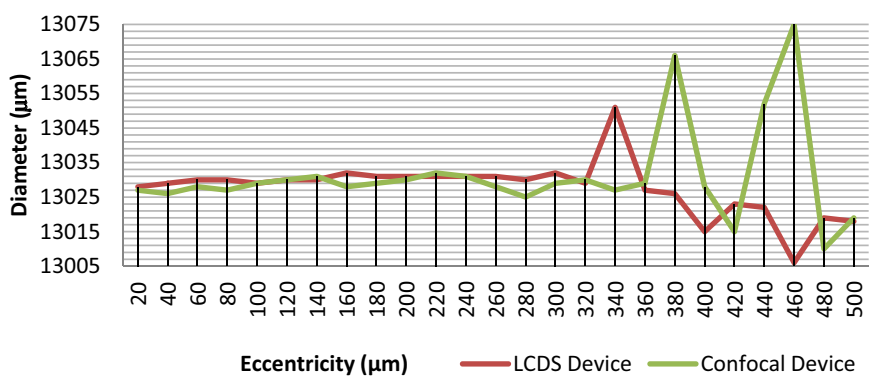

Fig. 5. Stability of readings with varying eccentricity.

This is done by first positioning the probe within $10 \mu \mathrm{m}$ of the hole centre. This initial positioning was achieved by bringing the probe inside the hole and manually adjusting the $\mathrm{X}-\mathrm{Y}$ table to align the probe with hole centre to within $10 \mu \mathrm{m}$. Then the probe is calibrated against the ring gauge. After calibration, a first reading of the diameter is taken. Then, the eccentricity was increased gradually in $20 \mu \mathrm{m}$ intervals before every reading until a significant variation in the measurements was observed. The result of eccentricity variations along X-Axis is shown in Figure 5.

Although Figure 5 presents results from one experiment, the effect on reading with varying eccentricity has been observed in several other experiments that showed the reading to be reliable within same ranges. The results show that diameter measurements of both devices remain stable within a couple of $\mu \mathrm{m}$ for small eccentricity values. After a point, larger variations can be observed which indicate that the devices are no longer providing reliable results. The LCDS device was able to give accurate reading up to an eccentricity of $320 \mu \mathrm{m}$ whilst the confocal device was accurate for up to $360 \mu \mathrm{m}$. Exceeding the above mentioned values of eccentricity, the readings varied randomly and become unreliable. Similar results were obtained for eccentricity along Y-axis.

On this basis, it was concluded that the probe in this particular experiment can safely measure accurately up to an eccentricity of $300 \mu \mathrm{m}$. This provides a reference eccentricity range within which the probe should be positioned during robotic measurement. It is important to 


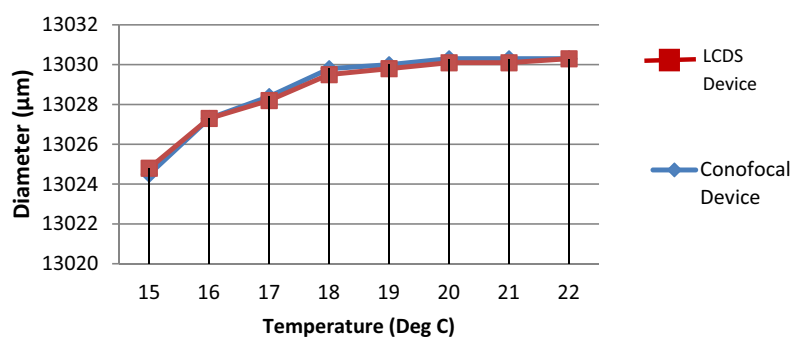

Fig. 6. Effect of thermal variations on diameter measurements.

mention here that the acceptable eccentricity values may wary for different materials and different surface finishes the study of which was beyond the scope of this paper. Moreover, for measuring deep hole, as the alignment of the probe in the hole becomes a major issues as the probe moves down the hole particularly for scanning hole geometry $[26,35]$. This can also exaggerate the eccentricity of probe. Deep hole measurement has detailed challenges that require extensive work which is beyond the scope of this paper. However, deep hole measurements and deep hole scanning is considered for future research work.

\subsection{Stage 1, Set 2: Effects of thermal variations and drift}

Researchers have explored the methods to understand and compensate for thermal variations in metrology [20,36,37]. In order to understand the effect thermal variations on the measurement accuracy, a simple experiment was conducted by systematically varying the temperature and recording the corresponding readings for the same artefact. Figure 6 shows the effect of thermal variations in this experiment.

As the temperature is increased from 15 to $19{ }^{\circ} \mathrm{C}$, the diameter measurement increased from just over $13024 \mu \mathrm{m}$ to a maximum of just over $13030 \mu \mathrm{m}$ (for Both devices). After initial rise in readings with temperature, the measurements appeared to settle at $13030 \mu \mathrm{m}$ (less than $1 \mu \mathrm{m}$ variation) from 18 to $21^{\circ} \mathrm{C}$. The stability in measurements could be the result of an equilibrium being established between expansion of measurement probe and the artefact. However, further investigation and detailed research is required to understand the effects of thermal variation on various materials in detail which was not in the scope of this paper. For this paper, it was decided to conduct the main set of experiments at temperatures between $18-21{ }^{\circ} \mathrm{C}$.

In addition to external temperature, internal temperature variation of an instrument can also cause measurements deviations in a phenomenon called "drift" [37,38]. In order to verify that thermal drift is not occurring, a range of measurements were taken over a period of one hour by keeping the external temperature constant. The results are presented in the form of a graph in Figure 7. As shown in the figure, only the LCDS device appear to have the issue of thermal drift where the readings keep on gradually decreasing (from 13026.8 to $13017.43 \mu \mathrm{m}$ )

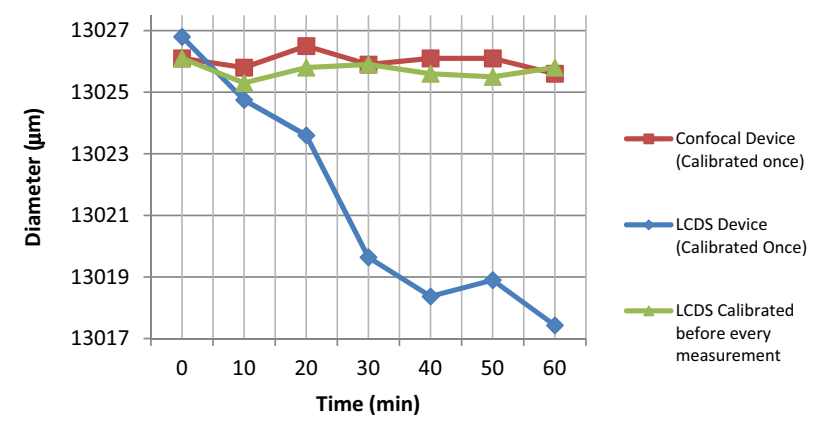

Fig. 7. Drift in measurement of confocal and LCDS devices.

with time. Thermal drift can occur in instruments due to internal heating. The normal trend of measurements due to thermal drift is downward as witnessed by the measurements from LCDS device (calibrated once). Devices are normally equipped with compensation coatings or algorithms to solve this issues which the LCDS device does not seem to be equipped with. The adopted solution to thermal Non-contact robotic hole measurement $17 \mathrm{drift}$ for LCDS device was to re-calibrate the probe before every measurement which made the readings stable as shown in Figure 7.

The confocal device on the other hand had no such issues and the readings were stable and consistent without any drift over the one hour period. Once the LCDS device was re-calibrated before every measurement, then both devices were found to be consistent for measurements with the variation only in the range of $1.5 \mu \mathrm{m}$ for diameter. Therefore, it was concluded that the confocal system had the ability to compensate for thermal drifts however the LCDS device should be calibrated before every measurement to provide the compensation. In the following experiments, the confocal device was only calibrated once whereas the LCDS device was calibrated before every measurement.

\subsection{Stage 1, Set 3: Effects of vibration on robotic measurements}

The vibrations on the robot have previously been studied for machining [1,39, 40] and assembly [41] involving micrometre accuracy. In an industrial scenario the servo motors of robots are in an ON-State that can create minor vibrations and subsequently affect the readings. An initial investigation on vibration is conducted to understand the range of vibrations involved in robotic hole measurement. For this purpose, robot vibrations were measured along the lateral axis of the hole. Understanding and interpreting vibrations required detailed investigation. Therefore, to get a generic understanding of the range of vibrations and their interpretation, the amplitude for vibrations was considered. Maximum displacement due to the vibrations during robotic measurement was recorded to be less than $0.2 \mu \mathrm{m}$. This range is acceptable for measurement within the application domain that this research is focused on. When measurements are taken at a rate much 
faster than the vibration frequency, it eliminates the effect of vibrations. Therefore, the effect of vibration was eliminated by setting the data collection rate to higher than vibration frequency. In this case $4 \mathrm{khz}$. Because the effect of vibration was found to be negilible on measurements, the effects of vibrations was not considered in the main set of experiments. However, further detailed investigations should be carried out to better understand the effects of vibration on measurement especially for measurement where the robot moves continuously during the measurements.

\subsection{Stage 2, Step 1: Accuracy and precision of robotic measurements}

To investigate the effects of robot movement on the measurement accuracy and precision, the designed artefact was first measured using the non-contact devices on a static platform and then on a robot. The static platform measurements provide a benchmark for robotic measurements. The robotic arm is moved through different routines before every measurement to mimic typical industrial activity. Movement of the robot is expected to introduce positional errors that could affect the measurement.

In an industrial scenario, a robot is normally in an "auto-mode" where the robot servos remain in their ONState. In this state, the position is maintained by the robot servo motors. This can create minor errors due to the motors' synchronisation. Therefore, as part of the experiment, the robot servo motors are kept in their ON-state during measurement. Measurements are also taken in "servos' OFF-State" when the position is maintained by mechanical brakes. Both these states have been considered to understand their effects on the measurements during various operational states of a robot. Brief description of the experiments is provided as follows.

1. Measurements on a static platform: the non-contact device was mounted on the angle plate. The probe was aligned to the central axis of the hole to be measured using level bar and shims for minor adjustments. The probe was centred for the ring gauge and the artefact's hole simultaneously using the XY-table. The probe was calibrated with the ring gauge. Then a set of measurements were taken for diameter, roundness, and surface $\mathrm{Ra}$ from the test artefact. In total sets of 20 readings were taken.

2. Taking measurements on robot with servos on/off: the non-contact device was mounted at the end of the robot arm. Similar to angle plate setup, the probe was aligned and centred for the artefact hole. The probe was then calibrated for hole measurement. The position of the robot was stored in the robot memory. A program was developed to move the robot along a preprogrammed path and bring it back to the recorded position. The pre-programmed path imitated the robot doing another inspection operation before coming back to measure the hole. The method of programming the robot was teach pendant programming. The robot was

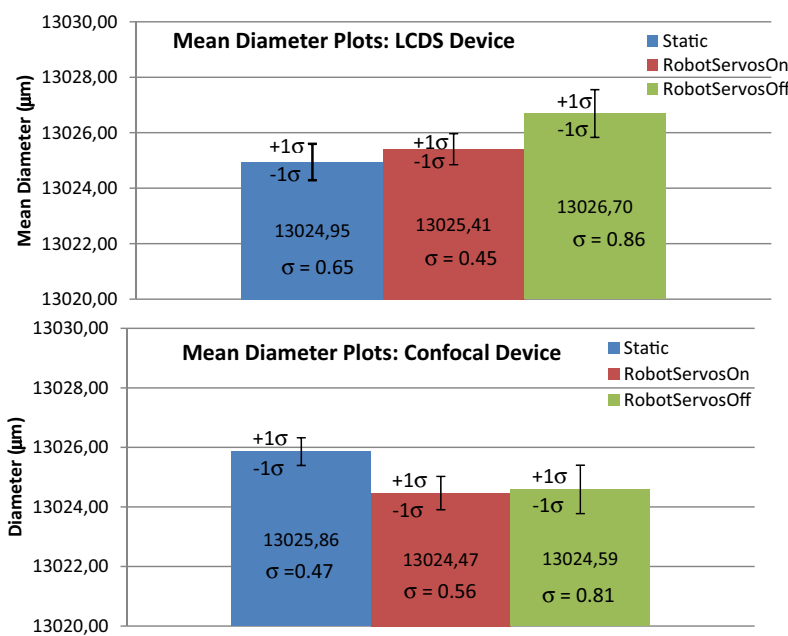

Fig. 8. Comparative plots of the mean hole diameter.

programed to move through a set of positions that covered the range of movement of the robot within the work cell. After each measurement the robot will come out of the hole, go towards the back of the cell, then move to the right side of the cell, then move back and forth imitating a measuremenmt and finally come back to the hole position where the prob can go back into the hoel for measurement. This wide range of movement can effect the measurements. Therefore, after each movement, a set of measurements for diameter, roundness and $\mathrm{Ra}$ of the hole were taken. The robot was moved through the programmed path before every measurement. After each cycle of movement, the robot servos were left $\mathrm{ON}$ during readings to imitate the actual behaviour of a robot used on an industrial context where robots mostly remain in their ON state. After the ON-state measurements, the robot servos were turned off to take measurements in an OFF-sate. Each parameter was measured 20 times. The mean values for diameters, roundness, roughness and their respective standard deviations $(\sigma)$ were calculated.

After collecting the data composed of readings of diameters, roundness, roughness ( $\mathrm{Ra}$ ) these measurements were statistically analyased to understand and interpret the accuracy and precision. For each type of measurement, the standard deviation and mean were calculated. The mean values of diameters, roundness, roughness ( $\mathrm{Ra}$ ) and their respective standard deviations $(\sigma)$ were plotted graphically to provide a comparison between readings taken on static platform and on robotic platform for the two noncontact hole measurement devices. In order to ensure the measurements are valid and reliable, the measurents on static platform were valided against measurement on a CMM. This was achieved by meauring the calibration ring at the same position on a CMM and with non-contact devices on static platform. The readings were in agreement between $2 \mu \mathrm{m}$. This provided the required to the measurement. This provided a basis for comparing measurements on static platform against the measurements on a robot. Figures 8-10 provide a comparison of readings between 
Table 1. Comparative statistical analysis of non-contact device on static platform and robot.

\begin{tabular}{cccccccc}
\hline & \multicolumn{2}{c}{ Diameter $(\mu \mathrm{m})$} & \multicolumn{2}{c}{ Roundness $(\mu \mathrm{m})$} & \multicolumn{2}{c}{ Roughness 'Ra' $(\mu \mathrm{m})$} & \\
& LCDS & Confocal & LCDS & Confocal & LCDS & Confocal & Static \\
Mean & 13024.95 & 13025.86 & 16.20 & 15.91 & 0.59 & - & Rlatform \\
\hline Std Deviation ' $\sigma$ ' & 0.65 & 0.47 & 0.26 & 0.05 & 0.06 & - & Robot \\
Std Deviation ' $\sigma$ ' & 13025.41 & 13024.47 & 16.05 & 16.15 & 0.63 & - & Servos-ON \\
\hline Mean & 0.45 & 0.56 & 0.35 & 0.07 & 0.05 & - & - \\
Std Deviation ' $\sigma$ & 13026.70 & 13024.59 & 16.02 & 16.14 & 0.58 & Robot \\
\hline
\end{tabular}

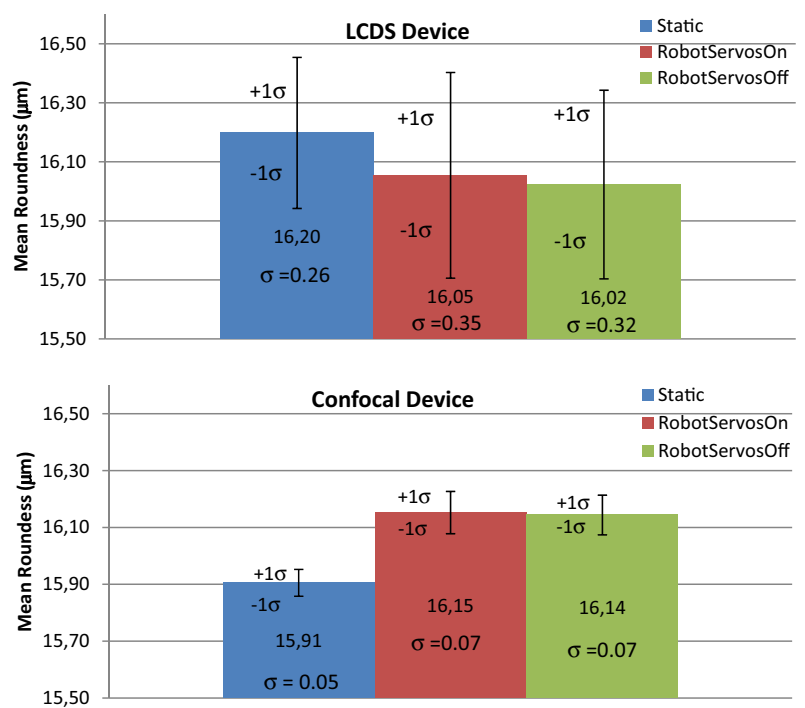

Fig. 9. Comparative plots for the mean hole roundness.

static platform and robot Because static platform is benchamrked against a CMM, a comparison of measurements on robot provides the basis for reliabilility of mesurements on robot. Figure 8 presents the comparative plots for mean hole diameters and its standard deviation. As shown in the figure, the mean readings of diameters vary slightly i.e. within $2 \mu \mathrm{m}$ for static and robotic platform for both the devices. It is also shown that the standard deviations are also similar. In general the standard deviation for the Confocal device is lesser than the LCDS device. Figure 9 shows comparative plot of mean hole roundness. The difference between roundness measurements for different platforms is within $0.10 \mu \mathrm{m}$ for the LCDS and within $0.12 \mu \mathrm{m}$ for the confocal device. The standard deviation for readings on LCDS varies from 0.26 to 0.35 from static to robotic platform. Similarly, the standard deviation for the Confocal device varies from 0.05 to 0.07 .

Figure 10 presents the comparative plots for the mean surface roughness $(\mathrm{Ra})$. This parameter is unique for the LCDS device as the confocal device cannot measure the Ra. This is one of the distinct and significant advantages of the non-contact measurement over conventional and other non-contact devices.

The grpah shows that the mean roughness varies from 0.59 to 0.63 from the static to robotic platform whereas the varies $\sigma$ varies from 0.06 to 0.05 . The range of variations in readings from the static platform to the robotic

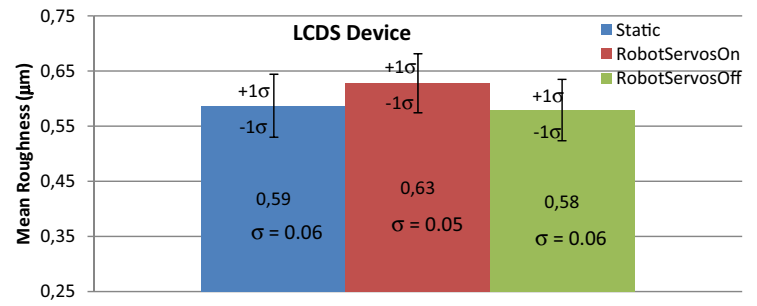

Fig. 10. Comparative plots for hole Roughness (Ra).

platform is negligible and the robot movement appears to have no significant effect on measurements.

An analysis and study of the graphs presented in Figures 8-10 shows that there is no significant difference between the readings taken on static platform and on the robot. The robotic measurements of diameters, roundness and Ra are very similar to the measurements on the angle plate. The range of variation is also very similar as evident from Table 1 which presents a summary of basic statistical analysis of the measurements. Table 1 shows that the standard deviations ' $\sigma$ ' for the readings of diameter on the robot are similar to the standard deviation on the static platform. For example, the $\sigma$ for LCDS device on robot with servos-On and Off are 0.45 and 0.86 respectively and the $\sigma$ for static platform it is 0.65 . Similar variation trends were found for roundness and $\mathrm{Ra}$ for both the devices as summarised in Table 1 . The variations in the readings from different platforms and devices are negligibly small which could have been caused by small difference in the position probes as a result of robot movement. These results verify that the robot movement and vibrations has no significant effect on the readings in these experiments.

\subsection{Stage 2, step2: ability to scan internal surfaces}

Scanning of hole surfaces can facilitate the detection of defects and surface anomalies. This ability gives non-contact measurement systems a distinct advantage over contact based system. For example, as shown Figure 10, the Ra value can be measured to $\mu \mathrm{m}$ accuracy using the LCDS device. Using the scanned data, internal hole features such the start point of taper and position of step in the holes can be measured. This section report the experiments conducted to scan the hole surfaces with the robotic measurement system to measure internal features. The features being measured are taper start point, taper end point, and the position of step in the hole. The LCDS and Confocal 


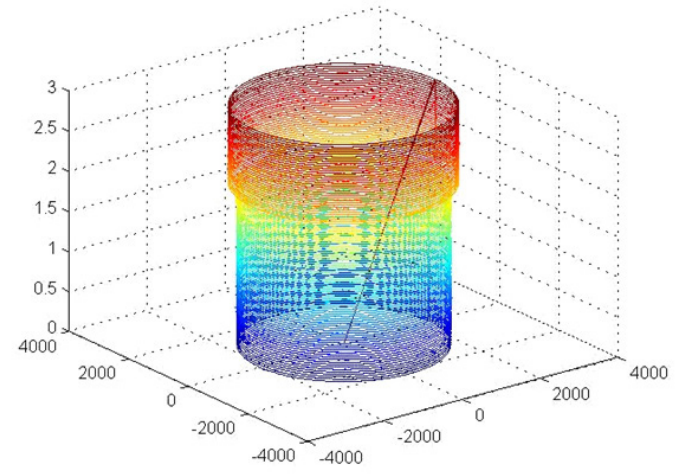

a) Stepped Hole Scan using Confocal Device

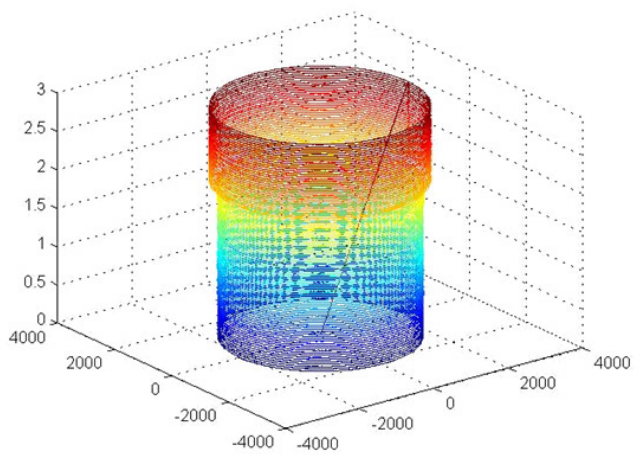

c) Stepped Hole Scan using LCDS Device

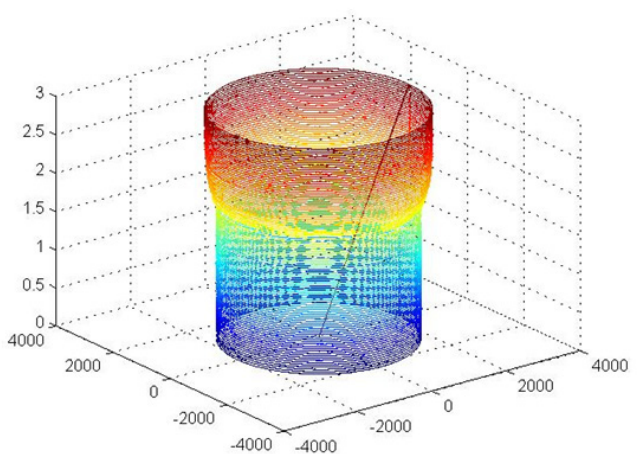

a) Tapered Hole Scan using Confocal Device

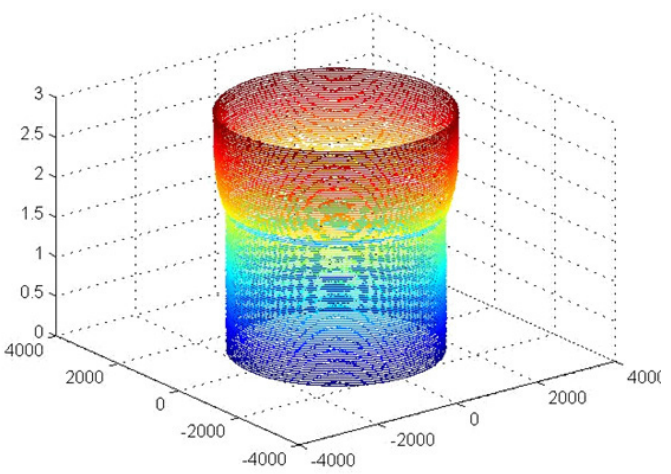

d) Tapered Hole Scan using LCDS Device

Fig. 11. 3D plot of the section containing taper and the section containing step using both the LCDS device and Confocal Device.

systems do not provide a measurement of thee parameters directly. However, these parameters can be extracted from the scanned data using tools such as Matlab.

In order to test the scanning of tapers and steps, both LCSD and Confocal devices were used to scan internal hole surfaces containing the tapers and steps. The scanning data was captured by bringing the probe to the hole centre and performing a scan by gradually moving the spinning probe down the hole. The scans were made in circular path of $30 \mu \mathrm{m}$ pitch over a section of $3 \mathrm{~mm}$. The sections containing the taper and the stepped hole were scanned. Figure 11 shows plots of the scanned tapered and stepped sections the artefact holes. As shown in Figure 11, both LCDS and Confocal devices can scan the holes and their hidden features i.e. tapers and steps.

To test the consistency of scans, a set of 20 scans were made for both the devices. In order to make the scans comparable, the same sections of the hole was scanned for both devices. The required parameters were then calculated by exporting and processing the data in Matalb. A simple method of using the rate of change of data was used to extract the relevant points from the data. Similar to the pervious experiments, the mean values and the standard deviation ' $\sigma$ ' of the parameters were calculated and plotted in in the form of bar charts as shown in Figure 12.

The data represented in Figure 12 shows that internal hole features can be measured using the non-contact devices with high consistency and that the robot movement has no effect on the ability to scan internal surfaces. The standard deviation for the confocal device appears to be marginally less than the LCDS device in determining hole features as shown in Figure 12. In terms of the quality of scans, both devices captured a reasonable amount of data with very little missing data. However, the confocal device had lesser missing data as compared to the LCDS device. For example, detailed attention to the scans presented in Figure 11 will show that the scans from the confocal device are denser as compared to the ones form the LCDS device. This perhaps also explains the reason before marginally smaller standard deviation in the readings from the confocal device.

The verification that it is feasible to scan internal hole surfaces and extract geometric information whilst a device is mounted on a robot provides a significant confirmation towards more wide spread use of robots for scanning and measuring challenging hole geometries and internal features.

\section{Conclusion and future research}

This paper has presented an investigation to understand the effect of robot movement on highly accurate and precise hole measurement. A contribution towards the use of robots for highly accurate and precise hole measurement has been made. 


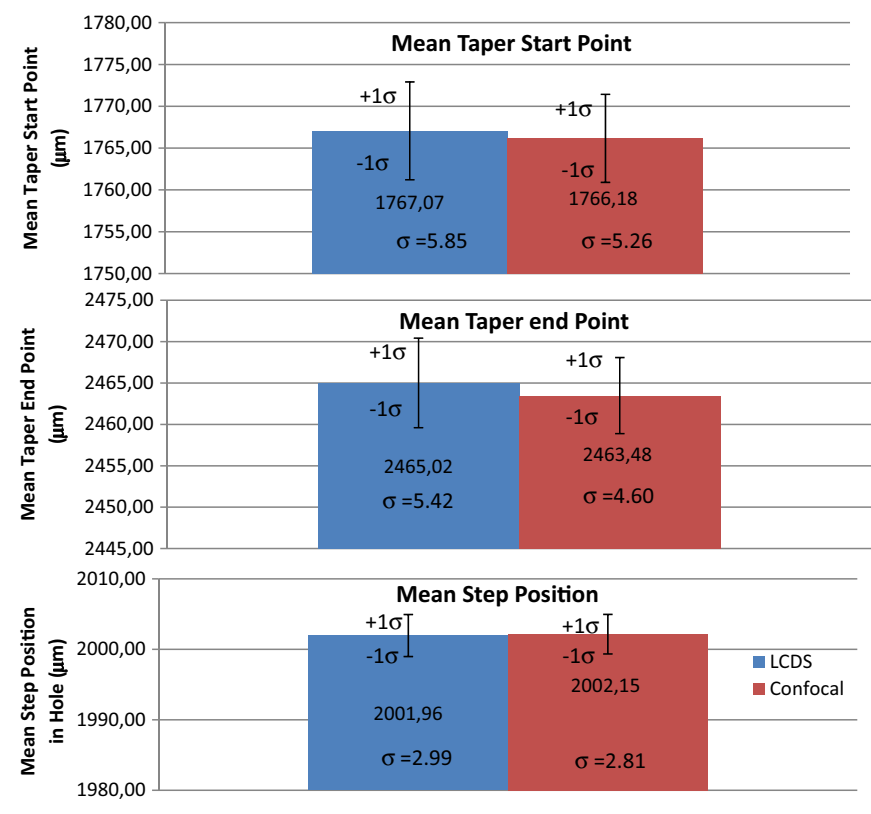

Fig. 12. Plots for taper start point, taper end point and hole's step position from the two devices.

A review of various hole measurement techniques and devices was conducted in the context of robotic metrology. Non-contact measurement methods were selected as the most promising for use with industrial robots. Two different non-contact devices i.e. the LCDS device and the confocal device were. The concept of manipulating highly accurate measurement devices using robotic arm was investigated. A set of experiments was performed to verify the impact of robotic manipulation on the accuracy of measuring the diameter, roundness, and surface roughness of holes. Each of the selected parameters was measured twenty times on a static platform and then on the robotic platform using both the devices. On the robotic platform, the robot was moved through a pre-programmed path before taking the measurements. The mean and standard deviation was calculated for each set of measurements. The results were plotted graphically to conduct a comparative analysis of the measurements. The results showed the measurements taken using the robotic platform were similar to the measurements taken on the static platform. It was shown from the results that the robotic manipulation has no significant effect on the accuracy and precision of measurement. This shows that the non-contact measurement devices can be used on the robot with the same confidence as on a static platform.

Before, the main set of experiments a set of enabling experiments were also conducted to understand the effects of thermal variations and vibrations on the measurements. The vibrations did not appear to have any noteworthy effect on the measurements. However, thermal variations appeared to have a minor effect on measurements. The measurements appear to be stable from $19{ }^{\circ} \mathrm{C}$ to $22^{\circ} \mathrm{C}$. Therefore, it was decided to conduct the main set of experiments at a temperature of $20-21{ }^{\circ} \mathrm{C}$. Moreover, thermal drift was also observed in the reading of the LCDS de- vice. A solution to this problem was regular calibration. Therefore, it was decided to calibrate the LCDS device before every measurement. The confocal device showed no thermal drift.

The experiments were extended to include the measurement of hidden internal features and parameters which are difficult to measure using traditional contactbased metrology equipment. Such features include the position of undercut and hidden steps and tapers in holes. The holes containing the taper and step were scanned several times whilst the devices were mounted on the robot. These experiments tested the effect of robotic movement on the consistency of scanning and the measurement of hidden parameters. The results showed the non-contact measurement devices can scan with high precision whilst being mounted on the robot. They also show that the variations introduced by the robot movement are negligibly small.

Overall, it can be stated that the non-contact measurement systems can measure with similar accuracy and precision on robots as on a static platform. The robot motion and vibration appear to have no significant effect on the measurements. It has been shown that the investigated non-contact measurement devices can be used on industrial robots for highly accurate and precise measurements of hole features.

For future work, an investigation should be carried out to understand the effects of robot movements on measurement when the measurements are taken during the robot motion. Moreover, the current non-contact hole measurement devices can only measure a small range of hole diameters. An integration and synchronisation the robot movement and the scanning probe should be investigated to increase the range of measureable diameters. This research should also involve the dynamic compensation of the effects of vibration and robot movement on the measurements. The future work should also explore the measurement of deep holes where the measurements will get effected as the probe moves down or up a deep hole.

\section{References}

1. C.-F. Bisu, M. Cherif, A. Gerard, J.-Y. K'Nevez, Dynamic behaviour analysis for a six axis industrial machining robot, Proc ICASAAM, September, 2011. Bucharest, Romania (Vol. abs/1201.4443) (2012)

2. J. Jamshidi, A. Kayani, P. Iravani, P.G. Maropoulos, M.D. Summmers, Manufacturing and assembly automation by integrated metrology systems for aircraft wing fabrication, Proc. Inst. Mech. Eng. Part B: J. Eng. Manuf. 224, 25-36 (2010)

3. M.H. To, A Framework for flexible integration in robotics and its applications for calibration and error compensation (Cranfield, Cranfield, 2012)

4. A. Weber, Virtual metrology and your technology watch list: ten things you should know about this emerging technology, Future Fab International 22, 52-54 (2007)

5. S. Lott, A robotic revolution, Aerospace Manufacturing Magazine (2011) 
6. B. Siciliano, O. Khatib, Handbook of Robotics (Springer, 2008)

7. J.L. Garbini, R.A. Saunders, J.E. Jorgensen, In-process drilled hole inspection for aerospace applications, Precis. Eng. 13, 125-134 (1991)

8. L.D. Peterson, S.J. Bullock, J.D. Hinkle, M.R. Hachkowski, P.A. Warren, M.S. Lake, Micron accurate deployable antenna and sensor technology for new-millennium-era spacecraft, in IEEE Proceeding of Aerospace Applications Conference, 1996, Vol. 1, pp. 129-139

9. M.S. Kumar, S.M. Kannan, Optimum manufacturing tolerance to selective assembly technique for different assembly specifications by using genetic algorithm, Int. J. Adv. Manuf. Technol. 32, 591-598 (2007)

10. R.S. Bunker, The effects of manufacturing tolerances on gas turbine cooling, J. Turbomachinery 131, 041011041018 (2009)

11. J. Liu, X. Pan, G. Wang, A. Chen, Design and accuracy analysis of pneumatic gauging for form error of spool valve inner hole, Flow Meas. Inst. 23, 26-32 (2012)

12. H. Kihlman, Affordable Automation for Airframe Assembly (Linköping University, 2005)

13. W. Cuypers, N. Van Gestel, A. Voet, J.P. Kruth, J. Mingneau, P. Bleys, Optical measurement techniques for mobile and large-scale dimensional metrology, Opt. Lasers Eng. 47, 292-300 (2009)

14. H. Nouira, R.H. Bergmans, A. Küng, H. Piree, R. Henselmans, H.A.M. Spaan, Ultra-high precision CMMs and their associated tactile or/and optical scanning probes, Int. J. Metrology Quality Eng. 5, DOI: 10.1051/ijmqe/2014009 (2015)

15. Nikon, Optical CMM Technology, http://www . nikonmetrology.com/en_EU/Products/PortableMeasuring/Optical-CMM/K-Series-Optical-CMM/ (brochure), accessed 11/01/2016 (2016)

16. Leica, Geosystem, Laser Tracker Systems, http://www. leica-geosystems.co.uk/en/Laser-Tracker-Systems_ 69045.htm, accessed 11/01/2016 (2016)

17. J.E. Muelaner, O.C. Martin, P.G. Maropoulos, Achieving Low Cost and High Quality Aero Structure Assembly through Integrated Digital Metrology Systems, Procedia CIRP 7, 688-693 (2013)

18. N. Jayaweera, P. Webb, Metrology-assisted robotic processing of aerospace applications, Robot. Comput. Integrated Manuf. 23 (2010)

19. G. Bone, D. Capson, Vision-guided fixtureless assembly of automotive components, Robot. Comput. Integr. Manuf. 19, 79-87 (2003)

20. S. Eastwood, P. Webb, Compensation of thermal deformation of a hybrid parallel kinematic machine (Pergamon Press, Inc., 2009), Vol. 25

21. Y. Bai, H. Zhuang, On the comparison of bilinear, cubic spline, and fuzzy interpolation Techniques for Robotic Position Measurements, IEEE Trans. Instrum. Meas. 54 (2005)

22. N. Calder, New dawns for robotics, Aerospace Manufacturing (2011)
23. Q. Yang, C. Butler, A 3-D noncontact trigger probe for coordinate measuring machines, Measurement 17, 39-44 (1996)

24. L.-M. Song, D.-P. Li, M.-C. Qin, Z.-Y. Li, Y.-l. Chang, J.T. Xi, Research on high-precision hole measurement based on robot vision method, Optoelectron. Lett. 10, 378-382 (2014)

25. Y. Zhao, P. Li, C. Wang, Z. Pu, A novel fiber-optic sensor used for small internal curved surface measurement, Sens. Actuat. A 86, 211-215 (2000)

26. M. Yu-zhen, Y. Yong-Xin, W. Xin-hua, Diameter measuring technique based on capacitive probe for deep hole or oblique hole monitoring, Measurement 47, 42-44 (2014)

27. G. Hui, Z. Xinglin, L. Shugui, S. Changku, Z. Yizhong, Measurement system of wall thickness of two adjacent blind holes, Chinese J. Sci. Instrum. (2003)

28. X. Hongji, W. Hongping, C. Guohua, D. Hongchang, A non-contact measurement method on size shape and position of deep cavity blind hole, in International Conference on Mechatronics and Automation (ICMA), 2003, pp. 546550

29. E.J. Chern, Non-contact eddy current hole eccentricity and diamatere measurement (1998)

30. R.L. Price, W.G. Jerome, Basic Confocal Microscopy (Springer, 2011)

31. Micro-Epsilon, Measurement Product Guide (2016)

32. P.M. Conn, Techniques in confocal microscopy (Elsevier, 2010)

33. A. Knuttel, Low-Coherence interferometric device for light-optical scanning of an object. United States (2005)

34. P. Godbillon, B. Lutat, A. Knuttel, Scanning sensor system for noncontact optical scanning of object surfaces (2010)

35. Z. Warlick, and P. Katz, Errors in non-contact sensor measurements due to misalignment and scanning methodology, Int. J. Metrolol. Quality Eng. 6 (2015)

36. D. Axinte, Miniaturised Robotic systems for holistic in-situ Repair and maintenance works in restrained and hazardous environments (University of Notttingham, Nottingham, 2013)

37. F. Marinello, M. Balcon, P. Schiavuta, S. Carmignato, E. Savio, Thermal drift study on different commercial scanning probe microscopes during the initial warming-up phase, Meas. Sci. Technol. 22, 094016 (2011)

38. ANSI/ISA-75.25.01, Test Procedure for Control Valve Response Measurement from Step Inputs (2000)

39. F. Girardin, Étude de l'usinage de matériaux performants et surveillance de l'usinage, L'Institut National de Sciences Appliquées de Lyon (2010)

40. D.A. Axinte, N. Gindy, K. Fox, I. Unanue, Process monitoring to assist the workpiece surface quality in machining, Int. J. Machine Tools Manuf. 44, 1091-1108 (2004)

41. R. Schmitt, M. Peterek, S. Quinders, Concept of a virtual metrology frame based on absolute interferometry for multi robotic assembly, in Precision Assembly Technologies and Systems, IFIP Advances in Information and Communication Technology (Springer, Berlin Heidelberg, 2014), Vol. 435, pp. 79-86 